\section{Cell cycle gene-specific control of transcription has a critical role in proliferation of primordial germ cells}

\author{
Daiji Okamura, ${ }^{1,2}$ Ikuma Maeda, ${ }^{1}$ \\ Hirofumi Taniguchi, ${ }^{1}$ Yuko Tokitake, ${ }^{1}$ \\ Makiko Ikeda, ${ }^{1}$ Keiko Ozato, ${ }^{3}$ Nathan Mise, ${ }^{4,7}$ \\ Kuniya Abe, ${ }^{4}$ Toshiaki Noce, ${ }^{5}$ \\ Juan Carlos Izpisua Belmonte, ${ }^{2,6,8}$ \\ and Yasuhisa Matsui ${ }^{1,8}$
}

${ }^{1}$ Cell Resource Center for Biomedical Research, Institute of Development, Aging, and Cancer, Tohoku University, Sendai, Miyagi 980-8575, Japan; ${ }^{2}$ Gene Expression Laboratory, Salk Institute for Biological Studies, La Jolla, California 92037, USA; ${ }^{3}$ Laboratory of Molecular Growth Regulation, National Institute of Child Health and Human Development, National Institute of Health, Bethesda, Maryland 20892, USA; ${ }^{4}$ Mammalian Cellular Dynamics, RIKEN BioResource Center, Tsukuba, Ibaraki 305-0074, Japan; ${ }^{5}$ Department of Physiology, School of Medicine, Keio University, Tokyo 113-0021, Japan; ${ }^{6}$ Center of Regenerative Medicine in Barcelona, Barcelona 08003, Spain

Transcription elongation is stimulated by positive transcription elongation factor $b$ (P-TEFb), for which activity is repressed in the 7SK small nuclear ribonucleoprotein (7SK snRNP) complex. We show here a critical role of 7SK snRNP in growth control of primordial germ cells (PGCs). The expression of $p 15^{I N K 4 b}$, a cyclin-dependent kinase inhibitor (CDKI) gene, in PGCs is selectively activated by $P$-TEFb and its recruiting molecule, Brd4, when the amount of active $P$-TEFb is increased due to reduction of the 7SK snRNP, and PGCs consequently undergo growth arrest. These results indicate that CDKI gene-specific control of transcription by 7SK snRNP plays a pivotal role in the maintenance of PGC proliferation.

Supplemental material is available for this article.

Received July 26, 2012; revised version accepted September 26, 2012.

Transcription is a highly regulated multistep process, and transcription elongation by RNA polymerase II (RNAPII) is a key step in the production of mature mRNA. Transcription elongation is stimulated by the positive transcription elongation factor $\mathrm{b}(\mathrm{P}-\mathrm{TEFb})$, and the 7SK small nuclear ribonucleoprotein (7SK snRNP) forms a complex

[Keywords: primordial germ cell; CDK inhibitor; transcription; $\mathrm{P}-\mathrm{TEFb}$; 7SK snRNP; Larp7]

${ }^{7}$ Present address: Division of Environmental Toxicology, Department of Pharmacology, School of Medicine, Jichi Medical University, Tochigi 329-0498, Japan

${ }^{8}$ Corresponding authors

E-mail belmonte@salk.edu

E-mail ymatsui@idac.tohoku.ac.jp

Article is online at http://www.genesdev.org/cgi/doi/10.1101/gad.202242.112. with P-TEFb to suppress P-TEFb activity (Diribarne and Bensaude 2009). Once the amount of any component of the 7SK snRNP (including Larp7) is reduced, the inhibitory complex becomes destabilized, and P-TEFb is released in an active form (Diribarne and Bensaude 2009). Recruiting molecules, such as Brd4, then recruit activated P-TEFb to the transcription start site (TSS) of target genes and phosphorylate Ser2 in the C-terminal domain (CTD) of RNAPII to stimulate transcription elongation (Jang et al. 2005; Yang et al. 2005). The 7SK snRNP is therefore considered as reservoir machinery to generally control transcription elongation.

Primordial germ cells (PGCs) are precursors of eggs and sperm and emerge as clusters of small numbers of cells in early embryos (Matsui and Okamura 2005; Hayashi et al. 2007; Saitou 2009). PGCs subsequently proliferate rapidly and increase in number but stop proliferation in later gestational stages in mammalian embryos (Western 2009). Male germ cells subsequently arrest in G1 phase until birth, while female germ cells immediately enter meiotic prophase. PGC proliferation is therefore dynamically regulated, but mechanisms underlying cell cycle progression of PGCs are not fully understood.

Eukaryotic cell cycle progression is tightly controlled by a number of molecular pathways. In particular, cyclins, cyclin-dependent kinases (CDKs), and CDK inhibitors (CDKIs) play pivotal roles in cell cycle control (Sherr and Roberts 1999). CDKIs belonging to the INK4 family, including $\mathrm{p} 15^{\mathrm{INK} 4 \mathrm{~b}}$, inhibit cyclinD-CDK4/6 and induce cell cycle arrest in G1 phase. Transcriptional regulation of CDKI expression has an important role in establishing G1 arrest under various biological conditions (Massague 2004). Previous studies indicate that expression of CDKI genes is upregulated in PGCs that have stopped proliferating (Western et al. 2008), suggesting their critical roles in regulation of PGC proliferation. In this study, we found that 7SK snRNPmediated selective repression of $p 15^{I N K 4 b}$ is necessary for PGCs to actively proliferate in mouse embryos.

\section{Results and Discussion}

We isolated Larp7, a gene encoding a La-related protein, and a component of the 7SK snRNP using differential cDNA screening because it was preferentially expressed in mouse PGCs (Supplemental Fig. S1A-F). We generated knockout mice lacking functional Larp7 to investigate Larp7 functions in PGCs (Supplemental Fig. S1G-I). Mice heterozygous for the Larp7 mutation were healthy and fertile. Homozygous mutant embryos were histologically normal (Supplemental Fig. S2C-S) but tended to be slightly smaller than their heterozygous mutant and wild-type littermates at embryonic day 16.5 (E16.5) (Supplemental Fig. S2A,B), and all homozygous mutants were, for unknown reasons, dead between E17.5 and the time of birth (Supplemental Fig. S3A). Because a few homozygous embryos were obtained at E19, it is likely that any pups that were actually born were eaten by their parents just after birth. A few Larp7 homozygous mutant embryos with a higher contribution of $129 \mathrm{~Sv}$ genetic background presented with a failure of neural tube closure (Supplemental Fig. S3B|, but this abnormality was not observed in Larp7 homozygous mutant embryos derived from backcrosses with C57BL/6 mice. 
We focused on PGC abnormalities in Larp7 homozygous mutant embryos. We first examined PGCs in mutant and wild-type embryos at E10.5 and found that the position and number of migrating PGCs in the homozygous mutants were indistinguishable from those in wildtype littermates (Supplemental Fig. S4A-E). We next examined PGCs in the embryonic gonads at E13.5; there were significantly fewer PGCs in male and female homozygous mutants than in wild-type embryos (Fig. 1A-E). These results indicate that Larp7 is necessary for PGCs to increase in number after E10.5.

We next assessed whether Larp7 functioned in the proliferation or survival of PGCs. Ratios of PGCs expressing phosphohistone $\mathrm{H} 3$ (pHH3), a marker for cells in G2-M phase of the cell cycle, and ratios of PGCs incorporating bromodeoxyuridine (BrdU), a marker of passage through $S$ phase, were lower in mutant embryos than in wild-type embryos (Fig. 1F,G; Supplemental Fig. S5A-X,a-h), but the ratios of active caspase 3-positive apoptotic PGCs in mutant embryos were not significantly different from those in wild-type embryos at E12.5 (Fig. 1H; Supplemental Fig. S5i-X). The number of germ cells in both males and females was still lower in homozygous mutant embryos
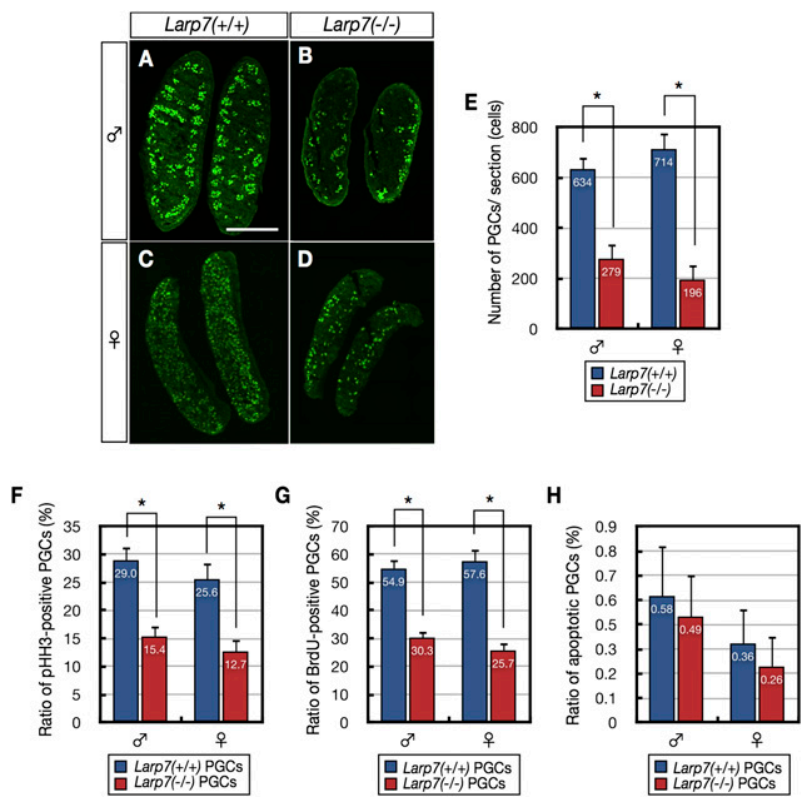

Figure 1. Reduced number of gonadal PGCs caused by the proliferation defect in Larp7 mutant embryos. $(A-D)$ PGC number in the E13.5 genital ridges of Larp7 mutant embryos $(B, D)$ and of wildtype littermates $(A, C)$ was estimated by detecting Stella-positive PGCs using immunostaining. (E) The average number of Stella-positive PGCs in the E13.5 genital ridge per section. PGCs in five sections from the middle part of individual genital ridges were counted, and the number of PGCs in three embryos of each genotype was determined. $(F-H)$ Loss of Larp7 function induced cell cycle arrest of PGCs in G1 phase but had no influence on apoptotic cell death of PGCs. The ratios of pHH3positive PGCs in G2/M phase $(F)$ and of PGCs incorporating BrdU during $S$ phase $(G)$ were determined using immunostaining of a frozen section of genital ridges from E12.5 embryos of each genotype. $(H)$ Apoptotic cells were detected by immunostaining for active caspase 3 . PGCs were identified by the expression of Oct $3 / 4(F, H)$ and by the expression of Stella $(G)$. $(F-H)$ A total of 1500-2500 PGCs in eight sections of both genital ridges of an embryo were counted, and ratios of the pHH3-positive, BrdU-positive, and active caspase 3-positive PGCs were determined for three embryos of each genotype; the average ratios are presented. $(E-G)\left(^{\star}\right) P<0.01$. Bar, $A-D, 0.3 \mathrm{~mm}$. than in wild-type embryos at E19 (Supplemental Fig. S4F$\mathrm{M})$. Taken together, these findings indicate that Larp7 functions in the proliferation and cell cycle progression of PGCs, most likely at the G1-S transition, but is dispensable for their survival.

We also examined differentiation of germ cells in the homozygous mutant embryos. TDRD1 (Chuma et al. 2003) and Dnmt3L (Sakai et al. 2004) are specifically expressed in male germ cells after E15.5 and E14.5, respectively, and the expression and localization of those proteins were normal in the homozygous mutant testis at E17.5 (Supplemental Fig. S6A-P). Scp3 is a component of the synaptonemal complex in meiotic germ cells and is specifically expressed and localized in the axial core in female germ cells after E14.5 (Yuan et al. 2000). Localization of Scp3 was normal in mutant germ cells in embryonic ovaries at E17.5 (Supplemental Fig. S6Q-X). Taken together, these findings demonstrate that differentiation of both male and female germ cells is not affected in the Larp7-deficient embryos, indicating that Larp7-mediated transcription control specifically regulates the cell cycle of PGCs. Although Larp7-deficient PGCs prematurely undergo growth arrest in genital ridges (Fig. 1), the expression of TDRD1 in male PGCs and of Scp3 in female PGCs is normally repressed until the correct time of their expression (Supplemental Fig. S6a-p), suggesting that differentiation of PGCs, including the initiation of meiosis in female germ cells, and their growth arrest are independently controlled.

We examined the functions of Larp7 in cell cycle control in PGCs in more detail using embryonic stem (ES) cellderived PGC-like cells in culture. We recently found that ES cells subjected to siRNA-mediated knockdown of the Max transcription factor (Hurlin and Huang 2006) are efficiently converted into PGC-like cells that express several germ cell-specific genes. We identified the PGC-like cells by monitoring expression of the Vasa-Venus transgene using flow cytometry; Vasa-Venus is specifically expressed in germ cells (Imamura et al. 2010). We introduced siRNAs targeting Larp7, 7SK methylphosphate capping enzyme (MePCE; another component of 7SK snRNP) (Jeronimo et al. 2007), or both, along with siRNA targeting Max into ES cells. The siRNAs for those and other related genes efficiently reduced their expression in ES cells and in Max knockdown cells (Supplemental Fig. S7). We analyzed the cell cycle of the PGC-like cells using flow cytometry (Fig. 2A). The number of cells in G1 phase was significantly increased, while the number in G2/M phase was apparently decreased, by knockdown of Larp7 or MePCE; simultaneous knockdown of Larp7 and MePCE resulted in additive effects (Fig. 2A; Xue et al. 2009). These results are consistent with the finding that the cell cycle in Larp7-deficient PGCs in embryos is abnormal (Fig. 1F,G). Moreover, these results indicate that 7SK snRNP positively controls the G1-S transition in PGCs. Larp7/MePCE knockdown in ES cells without Max knockdown resulted in an effect on the cell cycle (Supplemental Fig. S8) similar to that in Max knockdown ES cells (Fig. 2A), but the cell cycle profile of ES cells was apparently different from that of Max knockdown ES cells; the ratio of cells in G2 phase in Max knockdown cells (Fig. 2C) was substantially higher than that in ES cells, which resembled the cell cycle profile of PGCs (Seki et al. 2007), supporting the idea that the Max knockdown cells are a valid model to study cell cycle regulation in PGCs.

Because 7SK snRNP negatively regulates transcription, it is likely that 7SK snRNP suppressed transcription of 
A
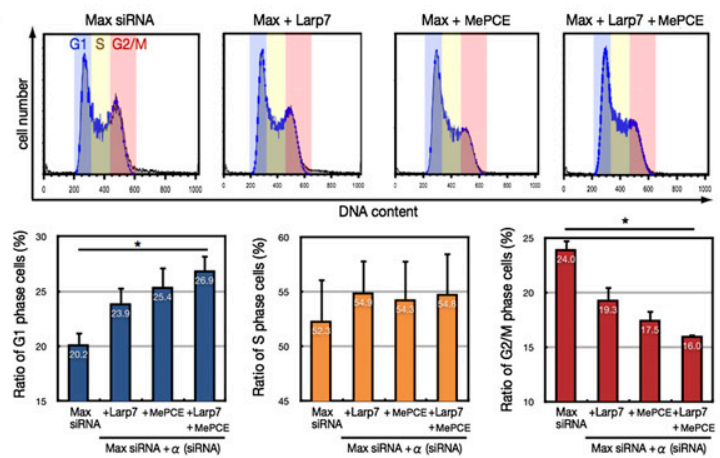

B
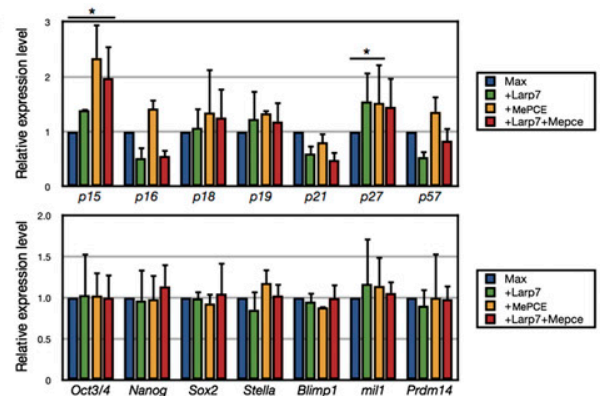

C
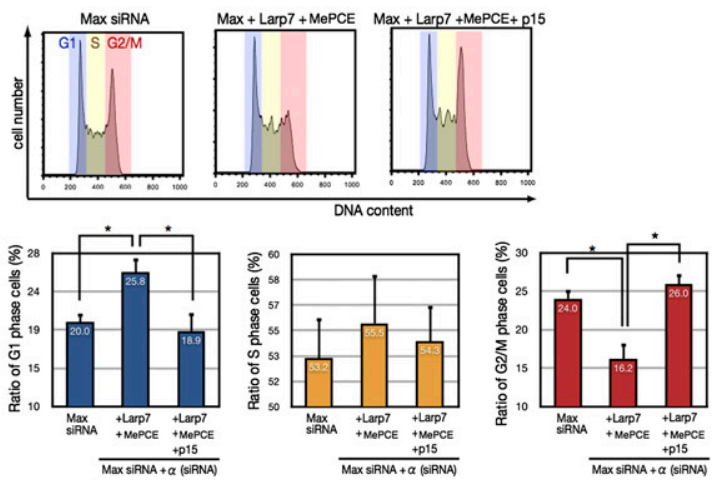

D
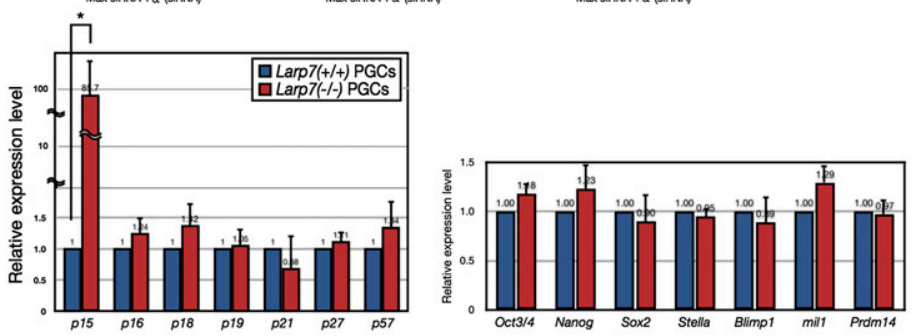

Figure 2. Induction of G1 arrest and CDKI gene expression in PGCs lacking Larp7, MePCE, or both. (A) The PGC-like cells were induced from the ES cells harboring the Vasa-Venus transgene by transfecting siRNA for $M a x$; in some ES cultures, siRNA for Larp7, MePCE, or Larp7 and MePCE were transfected along with Max siRNA. The cells were stained by propidium iodide (PI), and the cell cycle of the Vasa-Venus-positive PGC-like cells was analyzed using flow cytometry. (B) Quantitative RT-PCR was used to assess expression of the CDKI and germ cell-related genes in purified Vasa-Venus-positive PGC-like cells with or without knockdown of Larp7, MePCE, or both. The average values from three independent experiments are presented. $(C)$ Simultaneous p $15^{\text {INK4b }}$ knockdown in Larp7 and MePCE knockdown PGC-like cells rescued G1 arrest. $(A, C)$ In each experiment, 30,000 Vasa-Venus-positive cells from each sample were scored $(n=3)$. (D) Quantitative RT-PCR was used to assess expression of the CDKI and the germ cell-related genes in purified GFP-positive PGCs from Larp7 homozygous mutant and wild-type E12.5 embryos harboring the mill-GFP transgene. PGCs of three independent embryos of each genotype were used to estimate expression levels. $\left.{ }^{*}\right)$ $P<0.05(A-C) ;\left(^{\star}\right) P<0.01(D) .(A, B)$ Statistically significant differences between Max-only knockdown and other knockdown conditions are indicated.
CDKI genes that inhibited the G1-S transition in growing PGCs. We tested this hypothesis using the ES-derived PGC-like cells and found that expression of $p 15^{I N K 4 b}$ and $p 27^{\text {Kip } 1}$, as estimated by quantitative RT-PCR, was up-regulated by knockdown of Larp7, MepCE, and a combination of both (Fig. 2B). In contrast, expression of other CDKI genes as well as of a number of PGC-specific genes was not affected by knockdown of either gene or a combination of both, showing that 7SK snRNP selectively repressed expression of only some CDKI genes. In ES cells without Max knockdown, Larp7/ MePCE knockdown also induced expression of $p 15^{I N K 4 b}$, but instead of $p 27^{\text {Kip1 }}, p 21^{\text {Cip1 }}$ was additionally induced (Supplemental Fig. S9). To confirm the importance of increased $p 15^{\text {INK } 4 b}$ expression to inhibition of the G1-S transition in PGC-like cells, we determined whether the G1 arrest caused by Larp7/MePCE knockdown could be rescued by additionally introducing siRNA targeting p1 $15^{I N K 4 b}$; the proportions of PGC-like cells in G1 phase or G2/M phase were similar in cells treated simultaneously with four siRNAs $\left(p 15^{I N K 4 b}\right.$, Larp 7 , $M e P C E$, and $M a x)$ and those treated with only Max siRNA (Fig. 2C). We further investigated whether Larp7 deficiency and Larp7 siRNA caused the same abnormal gene expression in embryonic PGCs and PGC-like cells in culture, respectively. For this experiment, we purified mill-GFP-positive PGCs (Tanaka et al. 2004) from Larp7 homozygous mutant or wild-type embryos at E12.5. The results reveal that expression of $p 15^{I N K 4 b}$ is markedly higher in PGCs from homozygous embryos than in PGCs from wild-type embryos (Fig. 2D). Together, these results indicate that 7SK snRNP selectively represses expression of $p 15^{I N K 4 b}$ in growing PGCs, and when the function of 7SK snRNP is impaired by reduced expression of Larp7 or MePCE, more active P-TEFb may be preferentially recruited to the $p 15^{I N K 4 b}$ locus to activate transcription.

Our previous studies demonstrated that the bromodomain protein $\mathrm{Brd} 4$ associates with $\mathrm{P}-\mathrm{TEFb}$ to form an active complex, and this complex is recruited to TSSs of genes. We hypothesized that Brd4 was involved in the selective recruitment of active P-TEFb to $p 15^{I N K 4 b}$ in PGC-like cells lacking Larp7, MePCE, or both, and we assessed this possibility using a chromatin immunoprecipitation (ChIP) assay. We investigated binding of Cdk9, a subunit of P-TEFb (Peterlin and Price 2006), and of Brd4 to the TSS of $p 15^{I N K 4 b}$ in ES-derived PGClike cells. Binding of Cdk9 and Brd4 to $p 15^{I N K 4 b}$ was significantly increased after simultaneous knockdown of Larp7 and MePCE (Fig. 3A). In contrast, binding of Cdk9 to the TSS of Stella (Dppa3) or of Blimp1(Prdm1), representative PGCspecific genes (Saitou et al. 2002; Sato et al. 2002; Ohinata et al. 2005), was not changed in cells treated with Larp7 and MePCE siRNA. Moreover, Brd4 was not detected on the TSS of Stella(Dppa3) or of Blimp1(Prdm1) (Fig. 3A).

Because P-TEFb phosphorylates Ser2 in the CTD of the RNAPII large subunit, we examined changes in binding of the Ser2-phosphorylated 


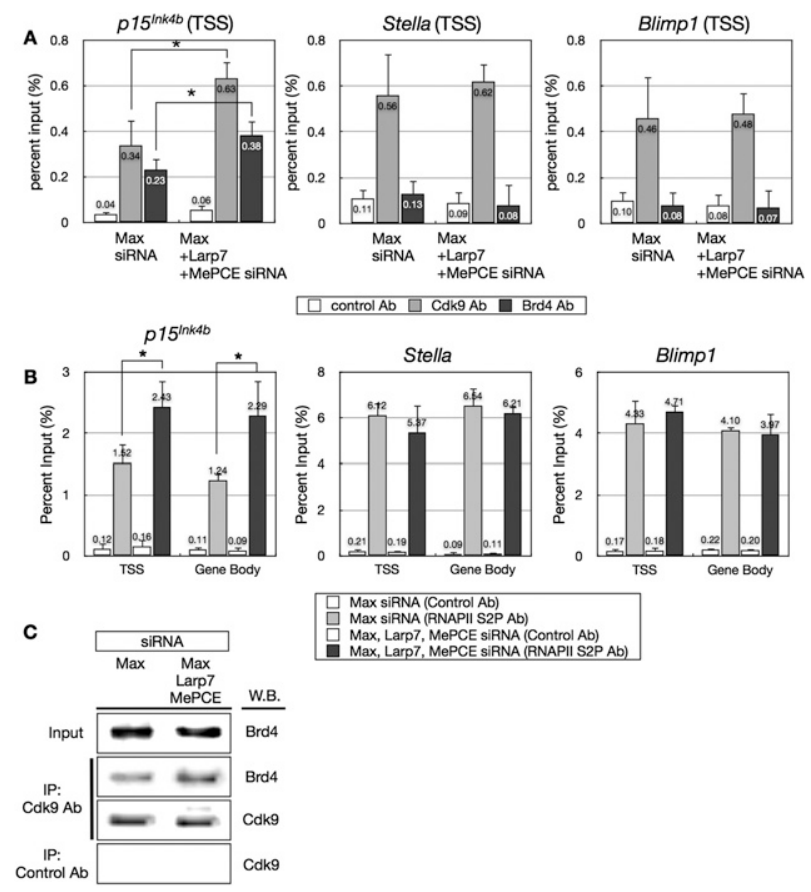

Figure 3. Selective increase of $\mathrm{Cdk} 9, \mathrm{Brd} 4$, and activated PNAPII binding at the TSS of $p 15^{I N K 4 b}$ in PGCs lacking Larp7, MePCE, or both. (A) ChIP analysis of Cdk9 and Brd4 binding at the p15 INK4b Stella, and Blimp1 TSSs in PGC-like cells and those lacking Larp7 and MePCE; Max siRNA induced the PGC-like state. The results of three independent experiments are presented. $\left({ }^{*}\right) P<0.05$. $(B)$ ChIP analysis of S2P-RNAPII (phosphorylation of Ser2 in the CTD of the largest subunit of the RNAPII enzyme) association at TSS and the gene body of $p 15^{I N K 4 b}$ (left), Stella (middle), and Blimp1 (right) in PGC-like cells and those lacking Larp7 and MePCE; Max siRNA induced the PGC-like state in ES cells. The results of three independent experiments are presented. $\left.{ }^{*}\right) P<0.05$. (C) Increased interaction of Cdk9 with Brd4 by knockdown of Larp7 and MePCE in PGC-like cells. Extracts of PGC-like cells with or without knockdown of Larp7 and MePCE were subjected to immunoprecipitation with control or anti-Cdk9 antibody and analyzed by immunoblot with the antibodies shown on the right. Essentially the same results were obtained by two independent experiments.

(S2P)-RNAPII to the TSSs as well as to the gene bodies of the genes by knockdown of Larp7 and MePCE by ChIP assay (Fig. 3B). Binding of S2P-RNAPII to the TSSs and the gene bodies of $p 15^{I N K} 4 b$ was significantly increased after simultaneous knockdown of Larp7 and MePCE in PGClike cells, but its binding to Stella and Blimp1 was not affected by knockdown (Fig. 3B). The results suggest that knockdown of Larp7 and MePCE results in the enhancement of phosphorylation of RNAPII and the subsequent stimulation of transcription elongation in $p 15^{I N K 4 b}$. We confirmed that the amount of Cdk9 associated with Brd4, which should be the active form, in PGC-like cells was increased by simultaneous treatment with Larp7 and MePCE siRNAs (Fig. 3C; Yang et al. 2008). Therefore, Brd4 most likely selectively recruited P-TEFb to $p 15^{I N K 4 b}$ in PGCs when the amount of active P-TEFb was increased due to repression of Larp7, MePCE, or both, and the recruited P-TEFb stimulated transcription elongation via phosphorylation of RNAPII. We confirmed the involvement of Brd4 in the activation of $p 15^{I N K 4 b}$ expression by additional knockdown of Brd4 in the Larp7/MepCE knockdown PGC-like cells and observed decreased expression of $p 15^{I N K 4 b}$ and decreased binding of Cdk9 to the TSS of $p 15^{I N K 4 b}$ by knockdown (Fig. 4A,B).

We finally indicated that more Cdk9 and Brd4 bound to $p 15^{I N K 4 b}$ in PGCs from Larp7-deficient embryos than those from heterozygous mutant embryos (Fig. 4C). Therefore, cell cycle progression in PGCs after E10.5 was ensured by 7SK snRNP-mediated repression of $p 15^{I N K 4 b}$.

As shown above, Brd4 selectively recruited active $\mathrm{P}$-TEFb to $p 15^{1 N K 4 b}$ when the amount of active P-TEFb was increased due to the reduction of Larp7 in mutant PGCs (Fig. 4C). Brd4 associates with acetylated histone (Dey et al. 2003), but its DNA sequence-specific binding is not known. It is therefore unclear how P-TEFb and Brd4 are preferentially recruited to $p 15^{I N K 4 b}$ in PGCs. To answer this question, we searched promoter regions of $p 15^{I N K 4 b}$, Stella, and Blimp1 and found that a conserved binding sequence of a transcription factor, IRF-1 (Miyamoto et al. 1988), existed only within the promoter region of $p 15^{I N K 4 b}$, and ChIP analysis indeed indicated specific binding of IRF-1 to $p 15^{I N K 4 b}$ in genital ridge cells (Supplemental Fig.
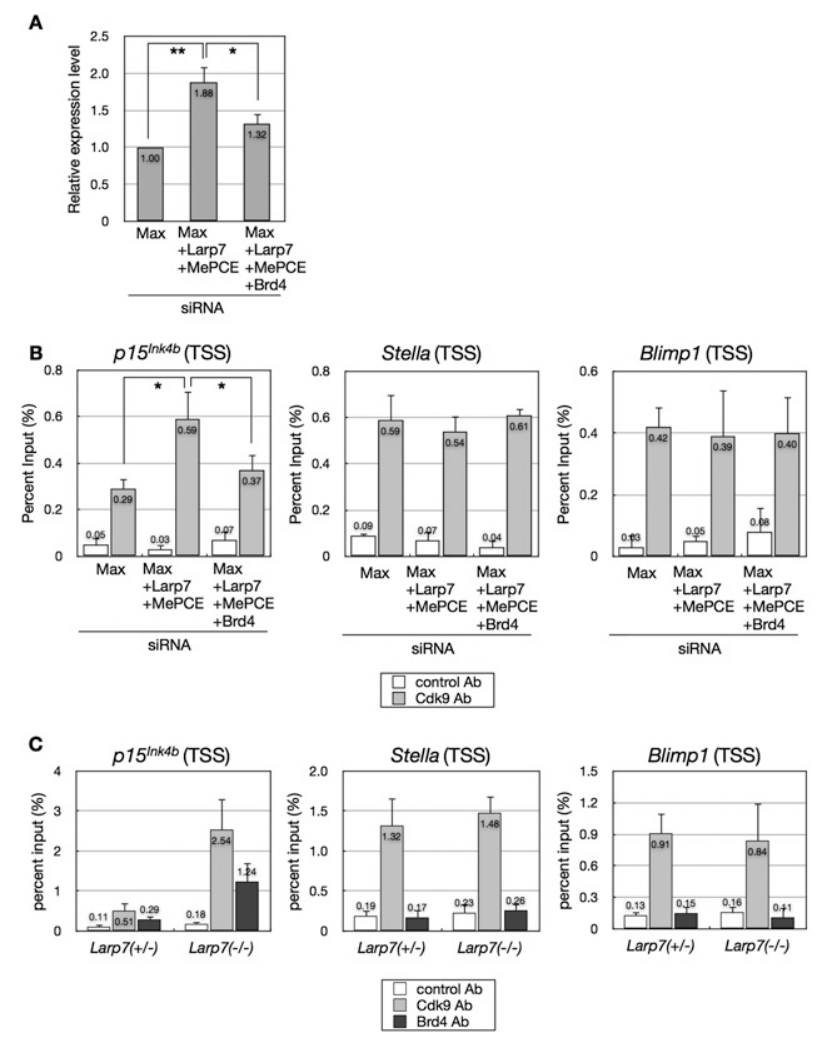

Figure 4. Involvement of Brd4 in the activation of $p 15^{I N K 4 b}(A, B)$ and the selective increase of Cdk9 and Brd4 binding at the TSS of p15 $5^{I N K 4 b}(C)$ in Larp7-deficient PGCs in vivo. $(A)$ Brd4 knockdown attenuated expression of $p 15^{I N K 4 b}$ induced by Larp7 and MePCE knockdown in PGC-like cells; Max siRNA induced the PGC-like state in ES cells. ES cells transfected with the indicated siRNA were applied for quantitative RT-PCR. The results of three independent experiments are presented. $\left(^{\star}\right) P<0.05 ;\left(^{\star \star}\right) P<0.01$. (B) ChIP analysis of Cdk9 binding at the $p 15^{I N K 4 b}$ (left), Stella (middle), and Blimp1 (right) TSSs in PGC-like cells and those lacking Larp7 and MePCE. The results of three independent experiments are presented. (*) $P<0.05$. (C) ChIP analysis of Cdk9 and Brd4 binding at the $p 15^{I N K 4 b}$, Stella, or Blimp1 TSSs in the genital ridge cells of E12.5 Larp7 homozygous mutant and heterozygous embryos. The results of two independent experiments are presented. 
S10A). IRF-1 was originally identified as a regulator of virus-inducible enhancer-like elements of the human INF-b gene and binds to a conserved sequence termed IRF-E (Tanaka et al. 1993). We then examined the association of Cdk9 and Brd4 with IRF-1 by a pull-down assay, and the result indicates that IRF-1 forms a complex with both Cdk9 and Brd4 (Supplemental Fig. S10B). We also found that additional knockdown of IRF-1 in Larp7/MepCE knockdown PGC-like cells resulted in decreased expression of $p 15^{I N K 4 b}$ (Supplemental Fig. S10C). Together, these results suggest that the association of Brd4 and P-TEFb with IRF-1 results in preferential binding of P-TEFb to $p 15^{I N K 4 b}$. We also found that IRF-1 is preferentially expressed in PGCs in embryos (Supplemental Fig. S10D,E).

7SK snRNP and Brd4 control transcription elongation in different kinds of cells (Wu and Chiang 2007; Barboric et al. 2009), but the phenotype of Larp7 mutant embryos revealed cell type-specific functions of 7SK snRNP in embryos. We investigated the expression of components of 7SK snRNP, Brd4, and IRF-1 in various embryonic tissues at E12.5. Although the expression of Hexim1, Hexim2, MePCE, and 7SK snRNA did not show apparent embryonic tissue specificity (Supplemental Fig. S11C-F), Larp7, Brd4, and IRF-1 were preferentially expressed in PGCs (Supplemental Figs. S11A,B, S10E), suggesting that selective expression of Larp7, Brd4, and IRF-1 contributed to the PGC-specific function of 7SK snRNP in embryos.

Our results reveal that the 7SK snRNP, in combination with Brd4, exerts gene-specific transcriptional regulation in PGCs via quantitative control of active P-TEFb (Supplemental Fig. S12), and this transcriptional regulation is critical for PGCs to actively proliferate. Previous studies indicate that Larp7 or the 7SK snRNP had anti-proliferative and tumor-suppressing functions in some kinds of cells, including hematopoietic cell lineage in Drosophila (Remillieux-Leschelle et al. 2002), cardiac myocytes in adult mice (Sano et al. 2002), and cultured cells (e.g., HeLa cells) (He et al. 2008). In addition, it was reported that Brd4 promotes cell cycle progression (Yang et al. 2008) and that Brd4 deficiency causes reduced proliferation of embryonic liver and lung cells (Houzelstein et al. 2002). In contrast, our results demonstrate that Larp7 has positive regulatory function in the cell cycle progression of PGCs (Figs. 1, 2) and that Brd4 positively controls $p 15^{I N K 4 b}$ expression (Figs. 3,4$)$. The discrepancy indicates that 7SK snRNP may show cell type-specific or developmental stage-dependent functions or both in transcriptional regulation, and these context-dependent functions may be controlled, at least in part, by sequence-specific transcription factors such as IRF-1 that may control preferential recruitment of $\mathrm{P}-\mathrm{TEFb}$ to specific target genes, as discussed above (Supplemental Figs. S10, S12).

Our results indicate that the functions of Larp7 on cell cycle regulation are restricted in gonadal PGCs because migrating PGCs in Larp7-deficient embryos are apparently normal (Supplemental Fig. S4), and the expression of Larp7 (Supplemental Fig. S1) and of other components of 7SK snRNP (data not shown) is up-regulated at E14 when PGCs undergo mitotic arrest. We speculate that additional $p 15^{I N K 4 b}$-specific transcription repressors and activators may ensure the active proliferation of early PGCs and the growth arrest of PGCs at E14. In summary, we demonstrated that the 7SK snRNP-Brd4-mediated regulation of $\mathrm{P}-\mathrm{TEFb}$ activity exerts cell type-specific and gene-specific control of transcription activation in mouse embryos and that this regulation plays a pivotal role in cell cycle progression in actively proliferating PGCs.

\section{Materials and methods}

Mouse strains and staging of embryos

Embryos were obtained from female mice of the outbred strain ICR that were mated with male BDF1 (B6D2F1) mice or with mill-GFP transgenic male mice (Tanaka et al. 2004), and Larp7 mutant embryos were obtained by intercrosses between Larp7 heterozygotes harboring the mill-GFP transgene.

\section{Targeted disruption of the Larp7 locus}

The Larp7 targeting vector (Supplemental Fig. S1G) was subjected to electroporation into the E14.1 ES cell line (129/Ola), which was injected into C57BL/6 blastocysts. We backcrossed the chimera mice and heterozygous mutant mice to $\mathrm{C} 57 \mathrm{BL} / 6$.

\section{Real-time PCR}

Real-time PCR was performed using gene-specific primer sets (Supplemental Table 1) or Power SYBR Green PCR master mix according to the manufacturer's instructions (Applied Biosystems).

\section{Assay for PGC proliferation}

PGC proliferation was assayed by pulse-labeling with BrdU as described (Seki et al. 2007) with minor modifications.

\section{Immunohistochemistry}

Immunohistochemical analyses with cryosections $(10 \mu \mathrm{m})$ were performed as described previously (Yamaguchi et al. 2005).

\section{RNAi}

VV3 ES cells harboring the Vasa-Venus transgene were transfected with siRNA oligonucleotides (Millipore) using Lipofectamine RNAiMAX (Invitrogen) according to the manufacturer's instructions.

Cell cycle analysis by fluorescence-activated cell sorting (FACS)

Dissociated ES cells were fixed in $1 \%$ paraformaldehyde, treated overnight in $70 \% \mathrm{EtOH}$, and then incubated with DNase-free RNase (Roche) and propidium iodide (Sigma).

\section{ChIP}

ChIP was performed using ES cells cultured for $72 \mathrm{~h}$ after siRNA transfection or dissociated genital ridge cells at E12.5 by the method described in the online protocol provided by Nippon Gene Co., Ltd. (http://www.nippongene.com/ pdf/manual/epigenetics/man_chip_reagents_090514.pdf).

\section{Statistical analysis}

Statistical differences were calculated using the Student's $t$-test. $P<0.05$ and $P<0.01$ were considered statistically significant.

The details of all experimental procedures are described in the Supplemental Material.

\section{Acknowledgments}

We are grateful to Dr. Anup Dey for the antibody recognizing Brd4, Dr. Shin-ichiro Chuma for the antibodies recognizing Scp3 and TDRD1, Dr. Shinya Yamanaka for the antibody recognizing Dnmt3L, and Dr. Takeshi Egawa for helpful advice on the experiments. Y.M. was supported by 
Grants-in-Aid for Scientific Research from the Ministry of Education, Culture, Sports, Science, and Technology of Japan.

\section{References}

Barboric M, Lenasi T, Chen H, Johansen EB, Guo S, Peterlin BM. 2009. 7SK snRNP/P-TEFb couples transcription elongation with alternative splicing and is essential for vertebrate development. Proc Natl Acad Sci 106: 7798-7803.

Chuma S, Hiyoshi M, Yamamoto A, Hosokawa M, Takamune K, Nakatsuji N. 2003. Mouse Tudor Repeat-1 (MTR-1) is a novel component of chromatoid bodies/nuages in male germ cells and forms a complex with snRNPs. Mech Dev 120: 979-990.

Dey A, Chitsaz F, Abbasi A, Misteli T, Ozato K. 2003. The double bromodomain protein Brd4 binds to acetylated chromatin during interphase and mitosis. Proc Natl Acad Sci 100: 8758-8763.

Diribarne G, Bensaude O. 2009. 7SK RNA, a non-coding RNA regulating P-TEFb, a general transcription factor. RNA Biol 6: 122-128.

Hayashi K, de Sousa Lopes SM, Surani MA. 2007. Germ cell specification in mice. Science 316: 394-396.

He N, Jahchan NS, Hong E, Li Q, Bayfield MA, Maraia RJ, Luo K, Zhou Q. 2008. A La-related protein modulates 7SK snRNP integrity to suppress $\mathrm{P}-\mathrm{TEFb}$-dependent transcriptional elongation and tumorigenesis. Mol Cell 29: 588-599.

Houzelstein D, Bullock SL, Lynch DE, Grigorieva EF, Wilson VA, Beddington RS. 2002. Growth and early postimplantation defects in mice deficient for the bromodomain-containing protein Brd4. Mol Cell Biol 22: 3794-3802.

Hurlin PJ, Huang J. 2006. The MAX-interacting transcription factor network. Semin Cancer Biol 16: 265-274.

Imamura M, Aoi T, Tokumasu A, Mise N, Abe K, Yamanaka S, Noce T. 2010. Induction of primordial germ cells from mouse induced pluripotent stem cells derived from adult hepatocytes. Mol Reprod Dev 77: 802-811.

Jang MK, Mochizuki K, Zhou M, Jeong HS, Brady JN, Ozato K. 2005. The bromodomain protein $\mathrm{Brd} 4$ is a positive regulatory component of $\mathrm{P}-\mathrm{TEFb}$ and stimulates RNA polymerase II-dependent transcription. Mol Cell 19: 523-534.

Jeronimo C, Forget D, Bouchard A, Li Q, Chua G, Poitras C, Therien C, Bergeron D, Bourassa S, Greenblatt J, et al. 2007. Systematic analysis of the protein interaction network for the human transcription machinery reveals the identity of the 7SK capping enzyme. Mol Cell 27: 262-274.

Massague J. 2004. G1 cell cycle control and cancer. Nature 432: 298-306.

Matsui Y, Okamura D. 2005. Mechanisms of germ-cell specification in mouse embryos. Bioessays 27: 136-143.

Miyamoto M, Fujita T, Kimura Y, Maruyama M, Harada H, Sudo Y, Miyata T, Taniguchi T. 1988. Regulated expression of a gene encoding a nuclear factor, IRF-1, that specifically binds to IFN- $\beta$ gene regulatory elements. Cell 54: 903-913.

Ohinata Y, Payer B, O'Carroll D, Ancelin K, Ono Y, Sano M, Barton SC, Obukhanych T, Nussenzweig M, Tarakhovsky A, et al. 2005. Blimp1 is a critical determinant of the germ cell lineage in mice. Nature 436: 207-213.

Peterlin BM, Price DH. 2006. Controlling the elongation phase of transcription with P-TEFb. Mol Cell 23: 297-305.

Remillieux-Leschelle N, Santamaria P, Randsholt NB. 2002. Regulation of larval hematopoiesis in Drosophila melanogaster: A role for the multi sex combs gene. Genetics 162: 1259-1274.

Saitou M. 2009. Germ cell specification in mice. Curr Opin Genet Dev 19: 386-395.

Saitou M, Barton SC, Surani MA. 2002. A molecular programme for the specification of germ cell fate in mice. Nature 418: 293-300.

Sakai Y, Suetake I, Shinozaki F, Yamashina S, Tajima S. 2004. Co-expression of de novo DNA methyltransferases Dnmt3a2 and Dnmt3L in gonocytes of mouse embryos. Gene Expr Patterns 5: 231-237.

Sano M, Abdellatif M, Oh H, Xie M, Bagella L, Giordano A, Michael LH, DeMayo FJ, Schneider MD. 2002. Activation and function of cyclin T-Cdk9 (positive transcription elongation factor-b) in cardiac musclecell hypertrophy. Nat Med 8: 1310-1317.

Sato M, Kimura T, Kurokawa K, Fujita Y, Abe K, Masuhara M, Yasunaga T, Ryo A, Yamamoto M, Nakano T. 2002. Identification of PGC7, a new gene expressed specifically in preimplantation embryos and germ cells. Mech Dev 113: 91-94.
Seki Y, Yamaji M, Yabuta Y, Sano M, Shigeta M, Matsui Y, Saga Y, Tachibana M, Shinkai Y, Saitou M. 2007. Cellular dynamics associated with the genome-wide epigenetic reprogramming in migrating primordial germ cells in mice. Development 134: 2627-2638.

Sherr CJ, Roberts JM. 1999. CDK inhibitors: Positive and negative regulators of G1-phase progression. Genes Dev 13: 1501-1512.

Tanaka N, Kawakami T, Taniguchi T. 1993. Recognition DNA sequences of interferon regulatory factor 1 (IRF-1) and IRF-2, regulators of cell growth and the interferon system. Mol Cell Biol 13: 4531-4538.

Tanaka SS, Nagamatsu G, Tokitake Y, Kasa M, Tam PP, Matsui Y. 2004. Regulation of expression of mouse interferon-induced transmembrane protein like gene-3, Ifitm3 (mil-1, fragilis), in germ cells. Dev Dyn 230: 651-659.

Western P. 2009. Foetal germ cells: Striking the balance between pluripotency and differentiation. Int I Dev Biol 53: 393-409.

Western PS, Miles DC, van den Bergen JA, Burton M, Sinclair AH. 2008. Dynamic regulation of mitotic arrest in fetal male germ cells. Stem Cells 26: 339-347.

Wu SY, Chiang CM. 2007. The double bromodomain-containing chromatin adaptor Brd4 and transcriptional regulation. I Biol Chem 282: 13141-13145.

Xue Y, Yang Z, Chen R, Zhou Q. 2009. A capping-independent function of MePCE in stabilizing 7SK snRNA and facilitating the assembly of 7SK snRNP. Nucleic Acids Res 38: 360-369.

Yamaguchi S, Kimura H, Tada M, Nakatsuji N, Tada T. 2005. Nanog expression in mouse germ cell development. Gene Expr Patterns 5: 639-646.

Yang Z, Yik JH, Chen R, He N, Jang MK, Ozato K, Zhou Q. 2005. Recruitment of P-TEFb for stimulation of transcriptional elongation by the bromodomain protein Brd4. Mol Cell 19: 535-545.

Yang Z, He N, Zhou Q. 2008. Brd4 recruits P-TEFb to chromosomes at late mitosis to promote G1 gene expression and cell cycle progression. Mol Cell Biol 28: 967-976.

Yuan L, Liu JG, Zhao J, Brundell E, Daneholt B, Hoog C. 2000. The murine SCP3 gene is required for synaptonemal complex assembly, chromosome synapsis, and male fertility. Mol Cell 5: 73-83. 


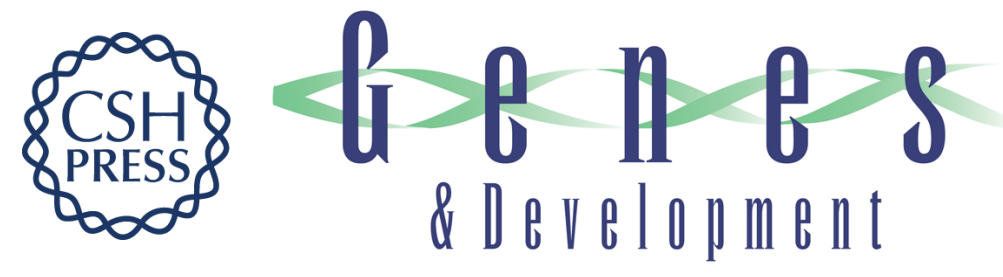

\section{Cell cycle gene-specific control of transcription has a critical role in proliferation of primordial germ cells}

Daiji Okamura, Ikuma Maeda, Hirofumi Taniguchi, et al.

Genes Dev. 2012, 26:

Access the most recent version at doi:10.1101/gad.202242.112

Supplemental http://genesdev.cshlp.org/content/suppl/2012/11/12/26.22.2477.DC1
Material

References This article cites 34 articles, 9 of which can be accessed free at:

http://genesdev.cshlp.org/content/26/22/2477.full.html\#ref-list-1

License

Email Alerting Receive free email alerts when new articles cite this article - sign up in the box at the top

Service right corner of the article or click here.

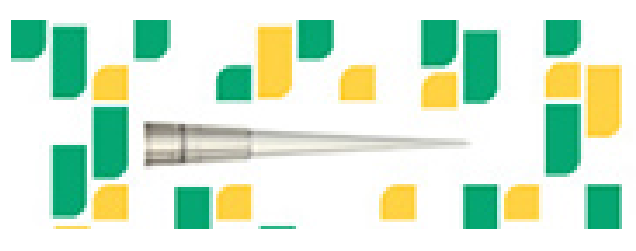

Focused on your science. 